\title{
Psychometric Properties Of The Persian Version Of The Uncivil Behavior In Clinical Nursing Education Among Nursing Students
}

This article was published in the following Dove Press journal:

Advances in Medical Education and Practice

\section{Tayebeh Hasan Tehrani ${ }^{1}$ Abbas Ebadi $\mathbb{1}^{2,3}$ \\ Zahra Mokhtari ${ }^{4}$ \\ Reza Ghanei Gheshlagh}

'Mother and Child Care Research Center, Hamadan University of Medical Sciences, Hamadan, Iran; ${ }^{2}$ Behavioral Sciences Research Center, Life Style Institute, Baqiyatallah University of Medical Sciences, Teheran, Iran; ${ }^{3}$ Nursing Faculty, Baqiyatallah University of Medical Sciences, Tehran, Iran; ${ }^{4}$ School of Nursing Midwifery, Tehran University of Medical Sciences, Tehran, Iran; ${ }^{5}$ Social Determinants of Health Research Center, Research Institute for Health Development, Kurdistan University of Medical Sciences, Sanandaj, Iran
Correspondence: Reza Ghanei Gheshlagh Gheshlagh Social Determinants of Health Research Center, Research Institute for Health Development, Kurdistan University of Medical Sciences, Sanandaj, Iran

Tel +98 9l 44050284

Email rezaghanei30@yahoo.com
Background: Some clinical staff nurses show unprofessional behavior toward nursing students in clinical nursing education that can negatively affect their self-esteem and professionally. Examination of uncivil behavior toward nursing students requires a valid and reliable instrument. Therefore, the goal of the present study was to examine the psychometric properties of the Persian version of the Uncivil Behavior in Clinical Nursing Education (UBCNE) among nursing students.

Methods: In this methodological study, a total of 558 nursing students participated who were selected using a convenience sampling method, and were asked to complete the Persian version of the UBCNE. The UBCNE was translated based on the WHO guidelines. The face, content, and construct (using explanatory and confirmatory factor analysis) validities were assessed. In addition, internal consistency was assessed using the McDonald's omega and the Cronbach's alpha coefficient, and stability was assessed using the test-retest method.

Results: Explanatory factor analysis led to two factors, including Dismissive Behavior and Exclusionary Behavior that together explained $62.1 \%$ of the total variance of uncivil behavior. In the confirmatory factor analysis, the comparative and parsimonious fit indices were very good, but the absolute fit indices were poor $(\mathrm{RMSEA}=0.116, \mathrm{GFI}=0.98, \mathrm{NFI}=0.97$, $\mathrm{AFGI}=0.83$, PNFI=0.78). Reliability of the UBCNE was found to be 0.93 and 0.97 , using the Cronbach's alpha coefficient and the test-retest method, respectively.

Conclusion: The two-factor structure of the UBCNE has good validity and reliability among nursing students. Therefore, it can be used to assess civil behavior in clinical nursing education.

Keywords: psychometric property, uncivil behavior, factor analysis, nursing student, Iran

\section{Introduction}

Clinical education is an important and potentially stressful aspect of nursing education, because it provides nursing students with the opportunity to use their knowledge. ${ }^{1,2}$ While clinical experiences provide student the opportunity to use their knowledge, stress is increased as they are applying it with real people, in real situations. ${ }^{3}$ The learning experiences of students in a clinical education have several aspects that are influenced by type of unit, clinical expectations, clinical supervisor, and clinical course outlines. In clinical education, nursing students are faced with many challenges in terms of relationship with other students, trainers, and staff nurses; these challenges form their professional and social character. ${ }^{4,5} \mathrm{~A}$ good relationship between nursing students and staff nurses can improve students' self-confidence, self-efficacy, 
motivation, and professional decision-making. ${ }^{6-8}$ Staff nurses are role-models for nursing students, and can facilitate their learning through adopting the training role in clinical education. ${ }^{9}$ However, clinical nurses sometimes exhibit hostile behavior toward nursing students in clinical education that can be a source of severe stress for students. ${ }^{10}$ Civil behavior focuses on respect for others, not disagreement, dispute, and debate. ${ }^{11}$ Clark believes that these uncivil behaviors can cause mental and physical disturbances to the people involved and may turn into threatening situations if neglected. ${ }^{12}$

Some previous studies have shown that uncivil behavior from staff nurses toward their students can create negative experiences for the students and impair their learning. ${ }^{1,13-15}$ Martini maintains that nursing students' dissatisfaction with clinical education is in part due to uncivil behavior from clinical staff nurses. ${ }^{16}$ An Australian study found that the relationship between staff nurses and nursing students was an important factor in determining the quality of students' experiences; because nursing students have less power in clinical settings, they are more vulnerable to uncivil behavior. ${ }^{17}$ Another study has shown that uncivil behavior in clinical education impairs staff nurses' ability to be professional, provide high-quality care, and pay enough attention to patients' health. ${ }^{18}$

The American nurses Association (ANA) code of ethics for nurses emphasizes the importance of creating an ethical environment and promoting a professional culture in order to respect human dignity, and regards such behaviors as belittling, harassment, and threatening as ethically unacceptable. ${ }^{19}$ Nurses' behavior as role-models for students in clinical education is of high importance, therefore, designing instruments to assess staff nurses' behavior should be a top priority. ${ }^{20}$ Assessing nurses' uncivil behavior can increase our understanding of this kind of behavior and help us design interventions to modify the nursing education environment. ${ }^{21}$

Most of the previous have been focused on mistreatment or uncivil behavior in nursing education, ${ }^{12,22,23}$ and few studies have examined different types of behavior in this setting. On the other hand, few qualitative studies have focused on incivility in clinical education using a specific instrument. ${ }^{1,24}$ Therefore, a valid and reliable instrument seems to be needed to measure this construct in nursing clinical education. Review of literature shows that some of the existing instruments assess uncivil behavior from physicians, coworkers, patients, or supervisors toward clinical nurses, ${ }^{25}$ uncivil behaviors from classmates, ${ }^{26}$ or uncivil behavior from the perspective of students and professors. ${ }^{12}$
One of the best instruments in this domain is the UBCNE developed by Anthony and Yastik (2014) using a qualitative study on nursing students' experiences involving nurses' uncivil behavior in clinical education. ${ }^{1}$ The interaction between nursing students and medical staff is stressful. ${ }^{27}$ Some evidence indicates uncivil behavior against nursing students. The first step in solving any problem is identifying the current situation, so this problem needs to be investigated and measured to take steps to reduce and control it. ${ }^{28}$

Nursing students are the future staff nurses and care providers that should be able to provide high-quality care in clinical settings. Hostile or dismissive behavior from staff nurses can make nursing students disappointed with clinical work that in turn can have negative implications for patients' health. Therefore, uncivil behavior in nursing clinical education should be assessed using valid and reliable instruments so that proper interventions can be designed to reduce it. Therefore, the present study is aimed at examining the psychometric properties of the Persian version of the UBCNE among nursing students.

\section{Methods Study Design}

This is a cross-sectional, methodological study that was conducted in 2016 for the translation and validation of the Uncivil Behavior in Clinical Nursing Education (UBCNE) among Iranian nursing students.

\section{Participants}

The sample included 250 nursing students in the Nursing and Midwifery School, Tehran University of Medical Sciences, who had received clinical education in hospital for at least one semester. The duration of the undergraduate nursing course in Iran is 8 semesters (4 years) and students enter the clinical courses from the second semester. Inclusion criteria were the willingness to participate in the study, a nursing student who passed one semester.

Sample size adequacy is an important issue in methodological studies, but there is not a definite idea about the proper sample size, and different guidelines have been proposed for it. The minimum sample size required for EFA is $3-10$ participants per item; we selected 20 participants per item (Overall, 250 participants). More details on this sample are provided in Table 1. Given that the minimum sample size required to perform confirmatory factor analysis is 200 , we selected 308 individuals via convenience sampling. ${ }^{29}$ 
Table I Mean And Standard Deviations Of Uncivil Behavior Scores By Demographic Variables

\begin{tabular}{|l|l|l|l|l|}
\hline \multicolumn{2}{|l|}{ Variable } & n & $\%$ & Mean \pm SD \\
\hline Gender & Male & 72 & 28.8 & $5.01 \pm 7.04$ \\
& Female & 178 & 71.2 & $7.51 \pm 9.93$ \\
\hline Semester & $2-4$ & 144 & 57.6 & $4.70 \pm 6.64$ \\
& $5-8$ & 106 & 42.4 & $9.09 \pm 10.68$ \\
\hline Presence of a trainer & Yes & 240 & 96 & $6.56 \pm 9.11$ \\
& No & 10 & 4 & $12.20 \pm 11.38$ \\
\hline Place of residence & Dormitory & 96 & 38.4 & $6.98 \pm 8.85$ \\
& Own house & 154 & 61.6 & $6.66 \pm 9.51$ \\
\hline
\end{tabular}

\section{Instrument}

The demographic information sheet and the UBCNE were used to gather data. In 2011, a qualitative study (with 4 focus groups) was conducted to determine the experiences of nursing students regarding Incivility in clinical education on 21 nursing students. Based on the results of this study, three themes of Exclusionary, hostile or rude, and dismissive were extracted. ${ }^{30} \mathrm{~A}$ few years later, the same research team based on these themes prepared 20 items about nursing students' Uncivil Behavior and examined its validity and reliability. The sample consisted of 118 nursing students. In the exploratory factor analysis, 12 items remained. These items were classified as Dismissive Behavior and Exclusionary Behavior. ${ }^{1}$ Higher scores mean more incivility. In that study, the reliability of the whole instrument based on Cronbach's alpha coefficient was 0.93 .

\section{Translation}

After obtaining permission from the original developers, the UBCNE was translated into Persian using the Forward and backward translation method, based on the WHO guidelines. ${ }^{31}$ The original version of the instrument was translated into Persian by two independent translators, and then the final Persian version was developed by the research team and back-translated into English by two other independent translators.

\section{Validity}

The validity of the UBCNE (with 12 items) was assessed using the face, content (qualitative), and construct validities. In order to examine face validity, the instrument was given to 10 nursing students, and they were asked to give feedback on the understandability of items. In order to assess content validity in a qualitative manner, the instrument was given to 10 nursing professors (5 clinical trainers and 5 faculty members), and they were asked to examine the items in terms of grammar, vocabulary, and placement. Finally the Persian version of the UBCNE was modified based on the recommendations.

Construct validity was evaluated using a maximum likelihood exploratory factor analysis (MLEFA) and confirmatory factor analysis (CFA). Sampling adequacy was assessed using the Kaiser-Meyer-Olkin (KMO) test, and the Bartlett's test of sphericity was used to examine the correlation matrix between the variables. KMO values between 0.7 and 0.8 are good and between 0.8 and 0.9 are excellent. ${ }^{32}$ Latent factors were extracted by MLEFA using varimax rotation with scree plot in SPSS version 16 .

A cut-off point of 0.40 was considered for factor loadings. In the next step, the extracted factors were examined using a CFA and the common goodness of fit indices, including the Chi-squared test of model fit, Root of Mean Square Error of Approximation (RMSEA), Comparative Fit Index (CFI), Normal Fit Index (NFI), Adjusted Goodness of Fit Index (AGFI), Parsimonious Normed Fit Index (PNFI), and chi-squared/degrees of freedom ratio (normalized chisquared CMIN/df); the indices were examined using LISREL 8.8. The acceptable thresholds for the goodness of fit indices and the results of CFA are presented in Table 3.

\section{Reliability Examination}

In order to examine reliability, internal consistency (using the Cronbach's alpha coefficient and the McDonald's Omega) and stability (using the test-retest technique) were assessed. The McDonald's Omega was calculated using the following formula: $\Omega=1-\left[\left[a-\sum h_{i}^{\prime}\right] /[a+2 b]\right] ; a$ is the number of items in the factor, $h_{i}^{\prime}$ is the total communality, and $b$ is the total loading for the items of the factor. The McDonald's Omega ranges from 0 to $1 .^{33,34}$

\section{Ethical Considerations}

Before starting the study process, the study objectives were explained to the participants and their written informed consents were obtained. In addition, the participants were reassured that their personal information remained confidential. This study was approved by the Ethics Committee of University of Social Welfare and Rehabilitation Sciences (approval number 98.801. T.1.21225) and conducted with permission from Tehran University of Medical Sciences and in accordance with the Helsinki Declaration. 


\section{Results}

The study sample included 250 undergraduate nursing students, including 178 women $(71.2 \%)$ and 72 men $(28.8 \%)$ with a mean age of $24 \pm 0.2$ years. Table 1 shows the means and standard deviations of uncivil behavior scores by demographic variables.

Face validity and qualitative content validity were confirmed according to the feedbacks from nursing students and qualified experts. At this point we've only rewritten a few times. Since the items were simple and easy to understand, there was no fundamental change in them.

\section{Exploratory Factor Analysis}

The sampling adequacy indices were in the acceptable range $(\mathrm{KMO}=0.923)$, a value of 0.8 or more was considered suitable for the factor analysis. ${ }^{35}$ The Bartlett's test of Sphericity was significant at $\mathrm{p}=0.0001$ ). The factor loadings extracted in the EFA were all above 0.40. In the EFA, 2 factors, including Dismissive Behavior (items 1, 2, 4, 7, 8, \& 11) and Exclusionary Behavior (items 3, 5, 6, 9, 10, \& 12) were extracted. As reported in Table 2 and Figure 1, two factors with eigenvalues of 6.954 and 1.219 , respectively, together explained $62.112 \%$ of the variance of uncivil behavior (Table 2 and Figure 1).

\section{Confirmatory Factor Analysis}

The study sample for CFA included 308 undergraduate nursing students, including 168 women (54.5\%) and 140 men $(45.5 \%)$ with a mean age of $23.3 \pm 3.2$ years. Most of the samples in the fourth academic year (33.7\%) and were resident in the dormitory (55.2\%). The chi-squared test of model fit $\left(X^{2}=447.31, \mathrm{p}=0.001\right)$ and the absolute, comparative, and parsimonious goodness of fit indices were examined in the EFA (Table 3). According to the results, the comparative and parsimonious fit indices were very good and the absolute fit indices were poor (Figure 2). We conducted confirmatory factor analysis on the UBCNE scale to test the fitness of the model obtained from exploratory factor analysis $\left(X^{2}=447.31\right.$, $\mathrm{df}=53, \mathrm{p}=0.001$ ). Indices $X^{2}$ P-value, RMSEA, CFI, NFI, AGFI, CMIN/df, and PNFI were used to check the fit of the model. To check the fit of the model, the indices should be closer to one. In this case, the model fits better and the data can better confirm the pattern of the assumed relationships. Further details are provided in Table 3.

As shown in Figure 2, all goodness-of-fit indices were satisfactory, which indicates the two-component model fit of the study data.

\section{Reliability}

The Cronbach's alpha coefficient and the McDonald's Omega were used to examine the reliability of the

Table 2 Factors Extracted For The UBCNE

\begin{tabular}{|c|c|c|c|c|}
\hline Factor & Items & $\begin{array}{l}\text { Factor } \\
\text { Loading }\end{array}$ & $\begin{array}{l}\% \text { Of } \\
\text { Variance }\end{array}$ & Eigenvalue \\
\hline \multirow[t]{2}{*}{$\begin{array}{l}\text { Dismissive } \\
\text { behavior }\end{array}$} & $\begin{array}{l}\text { I-Embarrassed you in the presence of others. } \\
\text { 4-Talked to you with an inappropriate tone. } \\
\text { 2-Did not care about you. } \\
\text { 8-Raised their voice when talking to you. } \\
\text { 7- Used snide remarks and sarcasm when speaking about musing students. } \\
\text { II-Called you incompetent. }\end{array}$ & $\begin{array}{l}0.838 \\
0.735 \\
0.705 \\
0.684 \\
0.622 \\
0.589\end{array}$ & 54.797 & 6.954 \\
\hline & \multicolumn{4}{|l|}{$\sum^{h} i=3.373, \mathrm{~b}=6.955$} \\
\hline \multirow[t]{2}{*}{$\begin{array}{l}\text { Exclusionary } \\
\text { behavior }\end{array}$} & $\begin{array}{l}\text { 9-Did not involve you in making care decisions, while they should have } \\
\text { involved you. } \\
\text { 10-Did not share with you patient information that you should have been } \\
\text { informed about. } \\
\text { 5-Refused to receive your report. } \\
\text { 12-Did not help you. } \\
\text { 6-Refused to give you a report. } \\
\text { 3-Gave you an incomplete report. }\end{array}$ & $\begin{array}{l}0.796 \\
0.791 \\
0.715 \\
0.639 \\
0.629 \\
0.586\end{array}$ & 7.323 & 1.219 \\
\hline & $\sum^{h} i=3.721, \mathrm{~b}=6.926$ & & & \\
\hline
\end{tabular}


Table 3 The Accepted Threshold Of Indexes And Fitting Of The Confirmatory Factor Analysis Model

\begin{tabular}{|l|l|l|}
\hline $\begin{array}{l}\text { Fitting } \\
\text { Indexes }\end{array}$ & Acceptable Range & $\begin{array}{l}\text { Our } \\
\text { Results }\end{array}$ \\
\hline$X^{2}$ P-value & $>0.05$ & 0.01 \\
RMSEA & Good $<0.08$, medium $<0.08$ to 0.1 , and & 0.116 \\
& weak $<0.1$ & \\
CFI & $>0.9$ & 0.98 \\
NFI & $>0.9$ & 0.97 \\
AGFI & $>0.8$ & 0.83 \\
CMIN/DF & Good $<3$ and acceptable $<5$ & 8.4 \\
PNFI & $>0.5$ & 0.78 \\
\hline
\end{tabular}

Abbreviations: RMSEA, root mean square error of approximation; CFI, comparative fit index; NFI, normed fit index; AGFl, adjusted goodness of fit index; CMIN/DF minimum discrepancy function by degrees of freedom divided; PNFI, parsimonious normed fit index.

instrument and each of its factors. A Cronbach's alpha of 0.930 was found for the total UBCNE and alphas of 0.901 and 0.897 were found for Dismissive Behavior and Exclusionary Behavior, respectively. In addition, the McDonald's Omega was found to be 0.785 and 0.841 for the two factors, respectively. Moreover, the intraclass correlation coefficient (ICC) was found to be 0.97 . Further details are provided in Table 4.

\section{Discussion}

The goal of the present study was to explore the psychometric properties of the Persian version of the Uncivil Behavior in Clinical Nursing Education (UBCNE). The UBCNE has been previously translated into Korean and validated in South Korea. ${ }^{24}$ The translated text was simple and understandable; therefore, no item was changed according to face and content validity examinations. In the EFA,

Scree Plot

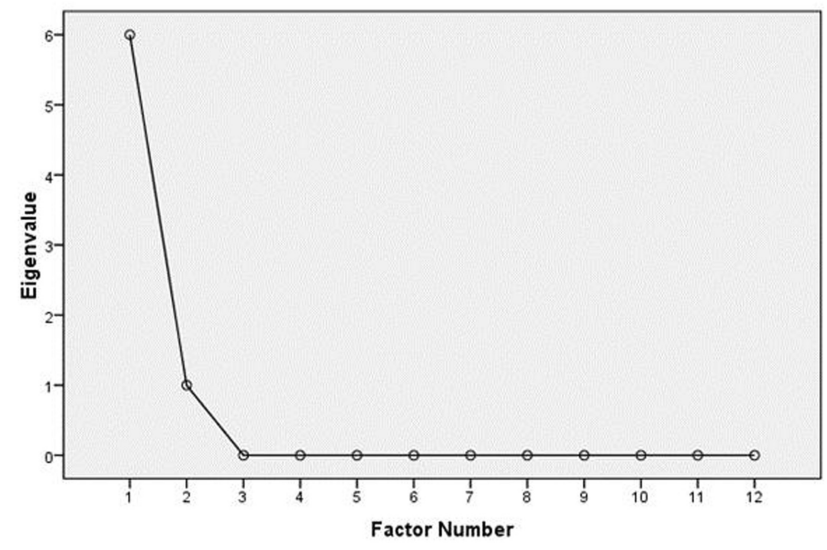

Figure I Scree plot for the extracted factors.

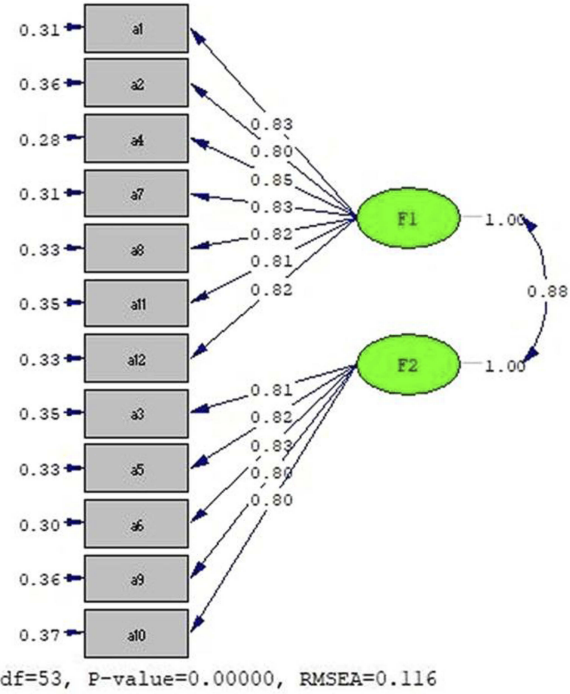

Figure 2 The final factor structure of the UBCNE.

Notes: FI, factor I (dismissive behavior); F2, factor 2 (exclusionary behavior).

the items assessing uncivil behavior were loaded on two factors: Dismissive Behavior (Items 1, 2, 4, 7, 8, \& 11) and Exclusionary Behavior (Items 3, 5, 6, 9, 10, \& 12) with eigenvalues of 6.954 and 1.219 , respectively. The two factors together explained $62.112 \%$ of the variance of staff nurses' uncivil behavior toward nursing students. In line with our findings, in the study by Anthony and Yastik, the items assessing uncivil behavior were loaded on two factors of Dismissive Behavior (Items 1, 2, 4, 7, 8, 11, \& 12) and Exclusionary Behavior (Items 3, 5, 6, 9, 10) with eigenvalues of 5.33 and 1.98, respectively, and together explained $60.90 \%$ of the variance of staff nurses' uncivil behavior toward nursing students. The placement of items on factors and the amount of variance explained in the original study are very similar to our findings, however, in the present study, a greater part of variance of Uncivil Behavior was explained, and item 12 (Did not help you) was loaded on Exclusionary Behavior, while in the original study it was loaded on Dismissive Behavior1 our finding seems to be more acceptable.

Jo and Oh (2016) validated the 20-item version of the UBCNE with three factors of Exclusion (items 2, 5, 7, 9, \& 10), Contempt (1, 2, 4, 6, and 14), and Refusal (12, 18, and 20) in South Korea. ${ }^{24}$ In this study, 7 items with low factor loadings were excluded and 13 items were kept, and the extracted factors together explained $49.61 \%$ of the total variance of Uncivil Behavior. Compared with this finding, a greater part of the variance of Uncivil Behavior was explained in the present study. Another difference is related to the factors extracted that can be 
Table 4 Results Of Examining The Internal Consistency And Stability Of The UBCNE

\begin{tabular}{|l|l|l|l|l|l|l|}
\hline \multirow{2}{*}{ Uncivil Behavior } & \multirow{2}{*}{ ICC (N=30) } & \multicolumn{2}{|l|}{$\mathbf{9 5 \%}$ Confidence Interval } & \multirow{2}{*}{ P-value } & Cronbach's & McDonald Omega \\
\cline { 3 - 5 } & & Lower Limit & Upper Limit & & Alpha & \\
\hline Factor I (dismissive behavior) & 0.927 & 0.846 & 0.934 & 0.001 & 0.901 & 0.785 \\
Factor 2 (exclusionary behavior) & 0.955 & 0.893 & 0.980 & 0.001 & 0.897 & $0.84 I$ \\
\hline
\end{tabular}

attributed to cultural differences or use of the 20 -item version of the instrument in the Jo and Oh's study. There are also differences between the two studies in the placement of items on factors. For example, the placement of items 5, 9, and 10 on Exclusionary Behavior and items 1, 2 , and 4 on Dismissive Behavior is similar in the two studies, but, in our study, item 12 was loaded on Exclusionary Behavior, while in the Jo and Oh's study, it was loaded on Dismissive Behavior. ${ }^{24}$ According to the results of CFA, the proposed model had a good fit to data. According to the results of CFA, the chi-squared test of model fit $\left(X^{2}=447.31, \mathrm{p}=0.001\right)$ and the absolute fit indices $(\mathrm{GFI}=0.88$ and $\mathrm{RMSEA}=0.116)$ were relatively poor, but the comparative $(\mathrm{CFI}=098),(\mathrm{IFI}=0.98$, and $\mathrm{NFI}=0.97)$ and parsimonious fit indices $(\mathrm{AGFI}=0.83$ and $\mathrm{PNFI}=0.78$ ) were in in a good range for the two-factor model.

Reliability is an index of how consistent an instrument is in its measurements. ${ }^{34}$ Using the Cronbach's alpha coefficient, the reliability of the total UBCNE and two factors of Dismissive Behavior and Exclusionary Behavior was found to be $0.930,0.901$, and 0.897 , respectively. In addition, the internal consistency of Dismissive Behavior and Exclusionary Behavior using the McDonald's Omega was found to be 0.785 and 0.841 , respectively that indicated good reliability of the instrument. In the original study, Cronbach's alphas of $0.93,0.86$, and 0.86 were reported for the total UBCNE, Dismissive Behavior, and Exclusionary Behavior, respectively; ${ }^{1}$ these findings are consistent with those of the previous study. In addition, Jo and Oh (2016) found an alpha of 0.84 for the total UBCNE, and alphas of $0.77,0.79$, and 0.68 for Exclusion, Contempt, and Refusal, respectively. ${ }^{20}$ The stability of the instrument was assessed using a test-retest method (two weeks interval) among 30 nursing students, and the scores obtained on the two administrations of the UBCNE were compared; a correlation of $\mathrm{r}=0.97$ was found. All of the main instrument items were culturally consistent with our culture, and we had no problem with cultural differences. This may be due to the simplicity of the items and the nature of the nursing profession.

\section{Conclusion}

Overall, it can be concluded that the Persian Version of the Uncivil Behavior in Clinical Nursing Education (UBCNE) can be used as a valid and reliable instrument to assess staff nurses' uncivil behavior toward nursing students during clinical education or internship in hospital wards.

\section{Acknowledgments}

The present study was funded by the University of Social Welfare and Rehabilitation Sciences (USWRS). The authors wish to thank the Vice-Chancellor of Research Affairs at the USWRS and all the students and professors who supported us in conducting this research.

\section{Disclosure}

The authors report no conflicts of interest in this work.

\section{References}

1. Anthony M, Yastik J, MacDonald DA, Marshall KA. Development and validation of a tool to measure incivility in clinical nursing education. J Prof Nurs. 2014;30(1):48-55. doi:10.1016/j.profnurs. 2012.12.011

2. Salminen L, Stolt M, Saarikoski M, Suikkala A, Vaartio H, LeinoKilpi H. Future challenges for nursing education-A European perspective. Nurse Educ Today. 2010;30(3):233-238. doi:10.1016/j. nedt.2009.11.004

3. Jamshidi N, Molazem Z, Sharif F, Torabizadeh C, Najafi Kalyani M. The challenges of nursing students in the clinical learning environment: a qualitative study. Sci World J. 2016;2016.

4. O’Mara L, McDonald J, Gillespie M, Brown H, Miles L. Challenging clinical learning environments: experiences of undergraduate nursing students. Nurse Educ Pract. 2014;14(2):208-213. doi:10.1016/j. nepr.2013.08.012

5. Billings DM, Halstead JA. Teaching in nursing-E-Book: A guide for faculty. Elsevier Health Sciences; 2015.

6. Levett-Jones T, Lathlean J, Higgins I, McMillan M. Development and psychometric testing of the belongingness scale-clinical placement experience: an international comparative study. Collegian. 2009;16 (3):153-162.

7. Hasanpour-Dehkordi A, Solati K. The efficacy of three learning methods collaborative, context-based learning and traditional, on learning, attitude and behaviour of undergraduate nursing students: integrating theory and practice. J Clin Diagn Res. 2016;10(4):VC01.

8. Hassanpour Dehkordi A, Heydarnejad MS. The effects of problembased learning and lecturing on the development of Iranian nursing students' critical thinking. Pak J Med Sci. 2008;24:5. 
9. Koontz AM, Mallory JL, Burns JA, Chapman S. Staff nurses and students: the good, the bad, and the ugly. Medsurg Nurs. 2010;19 (4):240.

10. Wallace L, Bourke MP, Tormoehlen LJ, Poe-Greskamp MV. Perceptions of clinical stress in baccalaureate nursing students. Int J Nurs Educ Scholarsh. 2015;12(1):91-98. doi:10.1515/ijnes-2014-0056

11. Clark CM, Carnosso J. Civility: a concept analysis. J Theory Constr Test. 2008;12(1):11.

12. Clark CM, Farnsworth J, Landrum RE. Development and description of the incivility in nursing education (INE) survey. $J$ Theory Constr Test. 2009;13(1):7.

13. Webster N, McKechnie JL. Webster's New Universal Unabridged Dictionary. Dorset \& Baber; 1983.

14. Hakojärvi H-R, Salminen L, Suhonen R. Health care students' personal experiences and coping with bullying in clinical training. Nurse Educ Today. 2014;34(1):138-144. doi:10.1016/j.nedt.2012.08.018

15. Felblinger DM. Incivility and bullying in the workplace and nurses' shame responses. J Obstetric Gynecolog Neonatal Nurs. 2008;37 (2):234-242. doi:10.1111/j.1552-6909.2008.00227.x

16. Marini ZA, Dane AV, Kennedy RE. Multiple Pathways to Bullying: Tailoring Educational Practices to Variations in Students' Temperament and Brain Function. In: The Developmental Relations among Mind, Brain and Education. Springer; 2010:257-291.

17. Levett-Jones T, Lathlean J, Higgins I, McMillan M. Staff-student relationships and their impact on nursing students' belongingness and learning. $J$ Adv Nurs. 2009;65(2):316-324. doi:10.1111/j.13652648.2008.04865.x

18. Khadjehturian RE. Stopping the culture of workplace incivility in nursing. Clin J Oncol Nurs. 2012;16(6):638. doi:10.1188/12.CJON.638-639

19. Association AN. Code of Ethics for Nurses with Interpretive Statements. Nursesbooks. org; 2001.

20. Tecza BM, Boots BK, Clay PM, et al. Development of an instrument to measure civil and uncivil behaviors in the hospital clinical environment: implications for nurse leaders. JNurs Admin. 2015;45(7/8):391397. doi:10.1097/NNA.0000000000000220

21. Clark CM, Nguyen DT, Barbosa-Leiker C. Student perceptions of stress, coping, relationships, and academic civility: a longitudinal study. Nurse Educ. 2014;39(4):170-174. doi:10.1097/NNE.0000000000000049

22. Afrazandeh SS, Pouraboli B, Sabzevari S. A survey on the educational citizenship behavior observance by nursing students. J Nurs Educ. 2017;6(1):9-15.
23. Del Prato D. Students' voices: the lived experience of faculty incivility as a barrier to professional formation in associate degree nursing education. Nurse Educ Today. 2013;33(3):286-290. doi:10.1016/j. nedt.2012.05.030

24. Jo SO, Oh J. Validity and reliability of the Korean Version of a tool to measure uncivil behavior in clinical nursing education. J Korean Acad Soc Nurs Educ. 2016;22(4):537-548. doi:10.5977/jkasne.2016.22.4.537

25. Guidroz AM, Burnfield-Geimer JL, Clark O, Schwetschenau HM, Jex SM. The nursing incivility scale: development and validation of an occupation-specific measure. J Nurs Meas. 2010;18(3):176. doi:10.1891/1061-3749.18.3.176

26. Cooper JR, Walker JT, Winters K, Williams PR, Askew R, Robinson JC. Nursing students' perceptions of bullying behaviours by classmates. Issues Educ Res. 2009;19(3):212-226.

27. Pecua SM Incivility: The Lived Experiences of Nursing Students. 2017.

28. Masoumpoor A, Abbaszadeh A, Rassouli M, Alavimajd H. Critical review of scales of incivility behaviors in nursing education. Med Ethics J. 2017;11(40):93-102. doi:10.21859/mej-114093

29. Plichta SB, Kelvin EA, Munro BH. Munro's Statistical Methods for Health Care Research. Wolters Kluwer Health/Lippincott Williams \& Wilkins; 2013.

30. Anthony M, Yastik J. Nursing students' experiences with incivility in clinical education. J Nurs Educ. 2011;50(3):140-144. doi:10.3928/ 01484834-20110131-04

31. Instruments. WPotaao. 2016; http://www.who.int/substance_abuse/ AfU. rtteAN.

32. Nikpour M, Tirgar A, Ebadi A, Ghaffari F, Firouzbakht M, Hajiahmadi M. Development and psychometric evaluation of a women shift workers' reproductive health questionnaire: study protocol for a sequential exploratory mixed-method study. Reprod Health. 2018;15(1):22. doi:10.1186/s12978-018-0456-0

33. Dunn TJ, Baguley T, Brunsden V. From alpha to omega: a practical solution to the pervasive problem of internal consistency estimation. Br J Psychol. 2014;105(3):399-412. doi:10.1111/bjop. 12046

34. Sharif Nia H, Akbar HA, Ebadi A, et al. Psychometric properties of the King Spiritual Intelligence Questionnaire (KSIQ) in physical veterans of Iran-Iraq Warfare. J Mil Med. 2015;17(3):145-153.

35. Mohammadinia L, Ebadi A, Malekafzali H, Allen KA, Nia HS. The design and psychometric evaluation of the Adolescents' Resilience in Disaster Tool (ARDT-Q37): a mixed method study. Heliyon. 2019;5 (7):e02019. doi:10.1016/j.heliyon.2019.e02019
Advances in Medical Education and Practice

\section{Publish your work in this journal}

Advances in Medical Education and Practice is an international, peerreviewed, open access journal that aims to present and publish research on Medical Education covering medical, dental, nursing and allied health care professional education. The journal covers undergraduate education, postgraduate training and continuing medical education including emerging trends and innovative models linking education, research, and health care services. The manuscript management system is completely online and includes a very quick and fair peer-review system. Visit http://www.dovepress.com/testimonials.php to read real quotes from published authors. 